\title{
Measuring Spatial Aggregation in Binary Epidemics: Correlative Analysis and the Advantage of Fractal-Based Sampling
}

\author{
Francis J. Ferrandino
}

Department of Plant Pathology and Ecology, The Connecticut Agricultural Experiment Station, P.O. Box 1106, New Haven 06504. Accepted for publication 24 June 2004.

\begin{abstract}
Ferrandino, F. J. 2004. Measuring spatial aggregation in binary epidemics: Correlative analysis and the advantage of fractal-based sampling. Phytopathology 94:1215-1227.

The incomplete sampling of a binary epidemic is nothing more than the overlap of two spatial patterns: the pattern of diseased plants and the pattern of sampled points. Thus, the information on the spatial arrangement of diseased plants obtained from such a sampling explicitly depends on the geometric locations of the sampled points. A number of procedures for sampling disease incidence are examined. These include samples placed on a regular grid, spatially clustered samples, randomly selected samples, and samples specified by a nested fractal design. The performance of these various sampling schemes was examined using simulated binary epidemics with varying degrees of spatial aggregation over different length
\end{abstract}

ABSTRACT

The characterization of plant disease in a field often relies on a limited number of samples of the plant population due to temporal and monetary constraints. Depending on the nature of the symptoms exhibited by the host-pathogen system involved and the goals of the epidemiologist, these samples can be taken in many ways. Disease evaluation can be based on a plant part (leaf, branch, stem, or root), a whole plant, or a pooled collection of plants (21). Disease expression can be binary (dead/alive, chlorotic/green, etc.), discrete (Horsfall-Barratt scale [16], other disease rating scales, etc.), or continuous (estimated percent severity, lesions per area). The size scale of the sample and the mode of evaluating disease can be mixed and matched at the discretion of the experimentalist. The resultant disease evaluations can be used to model disease spread in space, increase over time, and overall impact on final crop yields $(8-11,14,18,33)$.

For many "catastrophic" diseases like damping-off, the observed symptom is inherently binary ("dead" or "alive"). Alternatively, the size of a field and time constraints may force an epidemiologist to rate sample units as simply healthy or infected. In other cases, plant symptoms may be cryptic, requiring tissue samples be taken and analyzed in the laboratory. Such analyses often yield only the presence or absence of the pathogen in question (e.g., enzyme-linked immunosorbent assay, polymerase chain reaction analysis, antigen response, or isolation followed by taxonomic identification). The net result of sampling is a set of geographically placed "zeros" and "ones", corresponding to healthy and diseased sampling units, respectively. There are many instances where mapped binary disease data, as described previously, can be important. How does one analyze such a point-pattern and how does sample placement affect the outcome of the analysis?

Corresponding author: F. J. Ferrandino

E-mail address: Francis.Ferrandino@po.state.ct.us

Publication no. P-2004-0913-03R

(C) 2004 The American Phytopathological Society scales, generated using a Neyman-Scott cluster process. A modification of spatial correlation analysis specifically geared to binary epidemics is derived and shown to be equivalent to a $\chi^{2}$ test comparing the number of infected plant pairs to that expected from a spatially random epidemic. This analysis was applied to the data obtained using the various sampling schemes and the results are compared and contrasted. For the same number of sampling points, the fractal design is most efficient in the detection of contagion and provides spatial information over a larger range of distance scales than other sampling schemes. However, the regular grid sampling scheme consistently yielded an estimate of average disease incidence that had the smallest variance. Sampling patterns consisting of randomly selected points were intermediate in behavior between the two extremes.

Additional keywords: simulation, stochastic modeling.

Binary data are often pooled over relatively small areas to yield the fraction or count of plants that are dead within a certain sized quadrat. This process can be performed in the field and results in a discrete variable that can take on a finite number of values depending on the number of plants in the quadrat. The reliability of the resultant sample-based estimates of the population mean disease level will, of course, increase with increasing sample number. Estimated values of disease severity are often assumed to follow a known probability distribution such as the binomial or the Poisson for random processes, or the negative binomial (34) or the beta-binomial (19) when aggregation is important. The relation between the confidence intervals about the estimated level of disease and the number of samples is well known for these distributions $(19,22)$. A comparison of observed variance to the expected binomial variance can then lead to estimates of spatial aggregation via the intra-cluster or intra-quadrat correlation coefficient or other measures of contagion $(3,19,20,29)$. The fitted parameters for pooled data are strongly dependent on the details of pooling, i.e., quadrat size and the placement of quadrat boundaries by happenstance, both of which are arbitrary. The dependence of parameters on quadrat size, above, is due to the intrinsic connection between inter-quadrat variance and intraquadrat correlation $(3,20,23)$. For this reason, parameter changes caused by moving a sample unit within a quadrat are difficult to examine directly.

An alternative to quadrat methods is the 2DCORR approach involving the direct enumeration of sample pairs as a function of intersample distance $(12,13)$. The explicit treatment of geometry in this method provides a relatively straightforward evaluation of the effect of sample placement. In this paper, I present a radially symmetric version of $2 \mathrm{DCORR}$ designed to estimate spatial correlation as a function of intersample distance. As will be shown, this method has the advantage of being described by a $\chi^{2}$ distribution, so that confidence limits can be easily placed on model estimates of correlation. 
Ideally, the goal of sampling is to obtain maximum information with the fewest number of analyzed samples $(27,32)$. In order to do this, we must understand how sample placement affects the results of subsequent analysis. The purpose of this study is to assess the reliability of sample-based population estimates of a binary epidemic for different spatial sampling patterns. The population parameters to be estimated are mean disease level and the spatial correlation evaluated at various sample-sample distances. The reliability of these estimates will be determined as a function of sample number and sample placement.

\section{THEORY AND APPROACHES}

Sampling schemes. Suppose that $n$ sample points are to be located in a field of area $A$. The mean distance, $\lambda$, between nearest neighbor samples can be obtained by equally apportioning the total area among the $n$ sampling points and setting $\lambda$ equal to the diameter of a circle subtending the same area:

$$
\lambda=2 \cdot \sqrt{\frac{A}{(\pi n)}}
$$

A complete list of symbols used in this paper is given in Table 1. The simplest sampling scheme places points on a regular grid. This grid can be square, triangular, or hexagonal and is characterized by some minimal distance, $\lambda_{\min }$, between points in the sample (pages 318-319 in literature citation 4). The quantity $\lambda_{\min }$ is of the order of $\lambda$ and depends on sample geometry $\left(\lambda_{\min }=\right.$ $b \lambda ; b=1 / 2 \pi^{1 / 2} \approx 0.886$ for a square grid, $\pi^{1 / 2} / 3^{1 / 4} \approx 1.347$ for a triangular grid, and $2^{1 / 2} \pi^{1 / 2} / 3^{3 / 4} \approx 1.100$ for a hexagonal grid). No spatial information concerning distances smaller than $\lambda_{\text {min }}$ can be obtained from data sampled on a regular grid. Alternatively, a simple random sample can be used. In this case, each point is randomly selected from the entire field and the minimum distance between points in a point pair can be much smaller than $\lambda$.

Another sampling method involves the use of a cluster of sample points placed relatively close together, which is repeated throughout the field at a larger length scale (5). This hierarchical scheme provides information on a small distance scale, $\lambda_{w c}$ (within clusters), as well as at larger distance, $\lambda_{\mathrm{bc}}$, (between clusters). The above set of sample points are clustered in the geographical sense. Statistically, they are still treated as individual samples and not pooled as suggested by Cochran (3) and discussed by Hughes et al. (20).

As an example, consider a $160 \times 160$ square array of plants which are to be sampled at 441 locations. This can be accomplished by sampling the vertices of a $21 \times 21$ square grid with each square with eight plants on a side (Fig. 1, filled squares). Alternatively, a clustered sample can be constructed by locating 400 of the assay points on the vertices of 100 sampling squares, one plant spacing on a side $\left(\lambda_{\mathrm{wc}}: 1 \times 1\right)$, arranged in a $10 \times 10$ array of squares with $16\left(\lambda_{\mathrm{bc}}\right)$ on a side and the remaining 41

TABLE 1. List of symbols

\begin{tabular}{|c|c|c|}
\hline Parameter & Dimensions & Definition \\
\hline$<\cdot>$ & & Ensemble average of the variable "." \\
\hline "overbar" & & Signifies a spatial average \\
\hline$a$ & & Length scale for dispersal (equation A13) \\
\hline $\operatorname{cov}(\cdot, \cdot)$ & & Covariance of two variables \\
\hline$D(r)$ & {$[\text { distance }]^{-2}$} & Radial dispersal function describing the distribution of infected plants about a focus of infection \\
\hline $\operatorname{DE}(\alpha)$ & & $\begin{array}{l}\text { Detection efficiency }=\text { fraction of realizations for which nonrandom behavior is detected at a given } \alpha \text { level of } \\
\text { probability }\end{array}$ \\
\hline$E(\cdot), E_{H}(\cdot)$ & & Expectation value of the variable ".". $H$ subscript signifies the hypergeometric distribution \\
\hline$F$ & & Total number of disease foci \\
\hline$g(r), G(r)$ & & $\begin{array}{l}\text { Ripley's } g \text { function (29) equal to the probability that a plant a distance } r \text { from an infected plant is infected. The } \\
\text { capital letter signifies a result obtained from a spatial subsample rather than an ensemble average }\end{array}$ \\
\hline$H(i, n, I, N)$ & & $\begin{array}{l}\text { Hypergeometric distribution (equation 2): Probability of } i \text { infecteds within a sample of size } n \text { drawn from a popula- } \\
\text { tion of size } N \text { containing } I \text { infecteds }\end{array}$ \\
\hline$i$ & & Number of infected individuals in sample \\
\hline$i_{F}$ & & Number of infected individuals in a focus \\
\hline$I$ & & Total number of infected individuals in population \\
\hline$j, k$ & & Dummy integer variables \\
\hline$n$ & & Number of individuals in sample \\
\hline$N$ & & Total number of individuals in population \\
\hline$p$ & & Population-based probability of infection $(I / N)$ \\
\hline$p_{k \mid j}$ & & Conditional probability that $X_{k}=1$, given that $X_{j}=1$ \\
\hline$P(r)$ & & Probability of infection within a spatial subsample of the population \\
\hline$r$ & [distance] & Radial distance \\
\hline$\Delta r$ & [distance] & Width of window used to sum plant-pairs within a certain distance range. \\
\hline$r_{j k}$ & [distance] & Distance between point $j$ and point $k$ \\
\hline$r_{\chi}$ & [distance] & Distance at which equation A12 is a maximum \\
\hline$r_{\rho}$ & [distance] & Correlative length scale (equation 16) \\
\hline$s$ & & Disease severity within a focus \\
\hline$X_{k}$ & & Binary variable $(0=$ healthy, $1=$ infected $)$ indicating whether or not the $k$ th sample plant is infected \\
\hline$\aleph$ & & Intensity of focal aggregation (equation A19) \\
\hline$\lambda$ & [distance] & Characteristic length scale \\
\hline$\lambda_{\mathrm{wc}}$ & [distance] & Characteristic length scale within a cluster \\
\hline$\lambda_{\mathrm{bc}}$ & [distance] & Characteristic length scale between clusters \\
\hline$\sigma_{B}^{2}$ & & Variance in $i$ for the binomial distribution \\
\hline$\sigma^{2}{ }_{H}$ & & Variance in $i$ for the hypergeometric distribution \\
\hline$\sigma^{2}$ & & Observed variance in the variable "." \\
\hline$\sum_{i}^{2}$ & & Normalized variance (equation 6) \\
\hline$\mu_{X}$ & & Ensemble mean of the $X_{k}$ 's \\
\hline$\phi$ & & Number of total plant pairs \\
\hline$\phi_{i i}$ & & Number of doubly infected plant pairs \\
\hline$\Phi(r)$ & & Fraction of the total number of plant pairs less than or equal to a distance $r$ apart \\
\hline$\phi_{i h}$ & & Number of mixed-infected plant pairs \\
\hline$\rho$ & & Correlation coefficient \\
\hline
\end{tabular}


points are selected to fill out the boundary (Fig. 1, open circles, left panel). This cluster pattern can be generalized so that the inner square can be of any desired dimension less than the original eight plant spacings. A property and a potential problem with this approach is that all information about pairs of plants at the original length scale of eight plant spacings is lost. Thus, there is a trade-off of information from the original length scale to the smaller cluster length scale.

In one dimension, cyclic sampling using specifically chosen irregularly spaced sampling points has been shown $(1,17)$ to produce information on spatial correlation at all distance scales. The generalization of this approach to two dimensions is not trivial. However, the concept that unequally spaced sample points may yield data over a wider range of distance scale using the same number of samples is worthy of further investigation (2).

One sampling method, which mimics the behavior of cluster sampling but retains spatial information over a larger range of length scales, is based on a nested fractal design. One such design is illustrated in Figure 1 (open circles, right panel). Note that the 16 points of a $4 \times 4$ array are relocated to the vertices of five nested squares each one half as large as the previous one. Thus, in this case, length scales of $1,2,4,8$, and 16 are equally represented by the sides of the squares, each with six pairs (four sides plus two diagonals) per replicate pattern. This nested template is then repeated throughout the field producing a tiled pattern. The self-similar nature of this pattern ensures that length scales are very evenly represented for distances less than the tiling length scale (32 in this case) over which the pattern is repeated.

The distribution of sample point pair distances. Let $\phi(r)$ be defined as the number of unique sample point pairs which lie a distance of $r$ apart. For a set of $n$ sample points, there will be a total of $n(n-1) / 2$ unique sample point pairs. The number of plant pairs characterized by a certain length scale for all of the above sampling schemes is listed in Table 2. Due to the discrete nature of the sampling process, plots of $\phi(r)$ rapidly become complicated and uninformative. It is much more useful to plot the cumulative pair probability distribution, $\Phi(r)$, defined as the fraction of point pairs less than or equal to a distance, $r$, apart. The value of this function for each of the nested fractal, the random, and the $8 \times 8$ grid sampling schemes are plotted versus distance, $r$, in Figure 2. The inset graph in Figure 2 shows that, on the grand scale, the function $\Phi(r)$ is basically independent of sampling scheme and depends only on the shape and size of the sampled field. It is only at distances of the order of the tiling pattern (32 for the fractal) that sampling geometry has an effect. Note that $\Phi(r)$ for fractal sampling is almost a straight line for $r<28$, indicating a constant sampling fraction per distance over this range. The $\Phi(r)$ for the random sampling scheme, on the other hand, is roughly proportional to $r^{2}$ when $r$ is small compared with the size of the field. This corresponds to the fact that neighboring samples within a

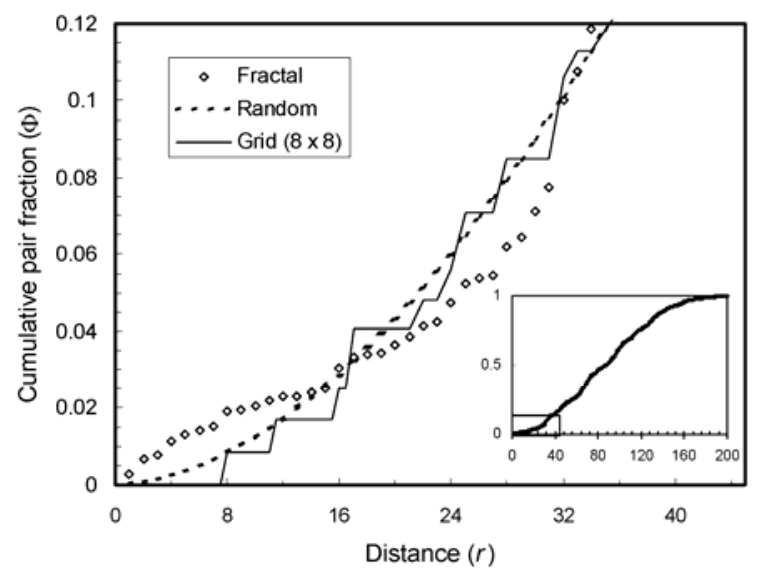

Fig. 2. Plot of the cumulative distribution function of plant pairs less than a certain distance apart. The fractal sampling scheme illustrated in Figure 1 (right panel) increases the number of pairs less than half the tile length apart $(<16)$ at the expense of intermediate distances $(16$ to 32$)$.
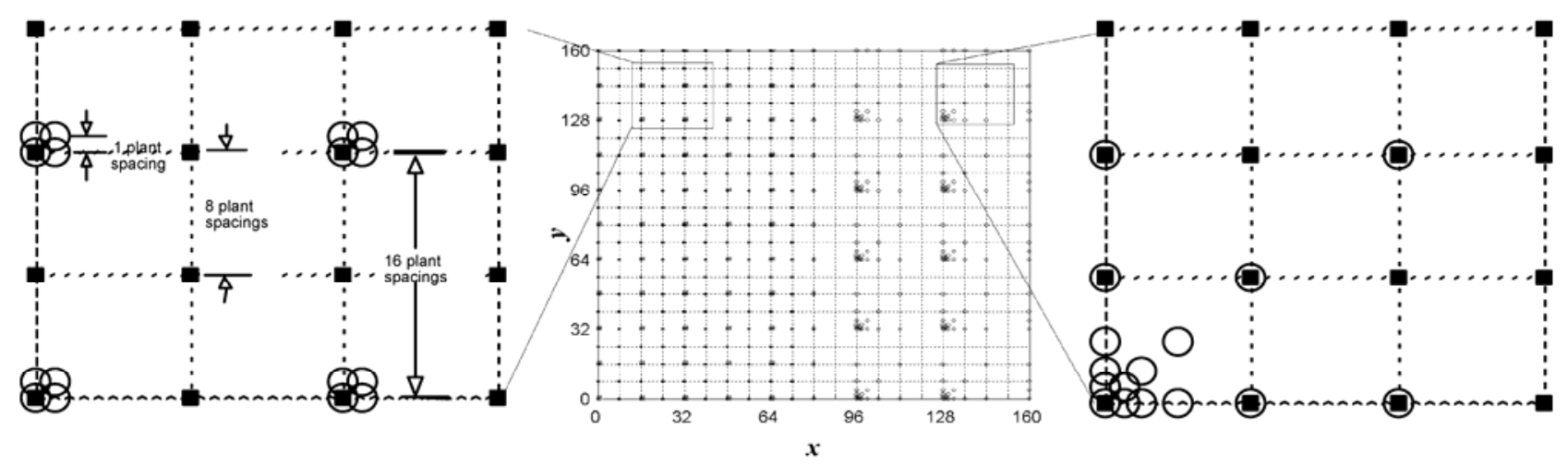

Fig. 1. The center panel illustrates three examples of sampling from a $160 \times 160$ grid of plants on a square lattice. The original regular sampling pattern (solid squares) lie on an $8 \times 8$ grid. The cluster sample is composed of four sample sites (open circles) on a $1 \times 1$ grid repeated on a $16 \times 16$ tiling pattern (enlarged in left panel). In the right panel, there is a comparison of the rectangular grid (filled squares) with a fifth-order nested fractal sampling scheme. Note that the assay points (open circles) lie on the vertices of five nested squares. The tiling length scale for this example is 32 units.

TABLE 2. A comparison of the number of plant pairs at various sampling length scales

\begin{tabular}{|c|c|c|c|c|c|c|c|}
\hline \multirow[b]{2}{*}{ Sampling scheme } & \multirow[b]{2}{*}{$\lambda_{\min }{ }^{\mathrm{a}}$} & \multicolumn{6}{|c|}{ Length scale } \\
\hline & & $\lambda<2$ & $2 \geq \lambda<4$ & $4 \geq \lambda<8$ & $8 \geq \lambda<16$ & $16 \geq \lambda<32$ & $32 \geq \lambda<64$ \\
\hline Random & 1 & 27 & 121 & 460 & 1,851 & 7,212 & 24,322 \\
\hline $1 \times 1$ cluster & 1 & 620 & 0 & 0 & 820 & 5,922 & 22,550 \\
\hline $4 \times 4$ cluster & 4 & 0 & 0 & 620 & 820 & 6,282 & 23,102 \\
\hline $8 \times 8$ grid & 8 & 0 & 0 & 0 & 1,640 & 6,604 & 24,084 \\
\hline Tiled fractal & 1 & 275 & 475 & 720 & 955 & 5,081 & 22,698 \\
\hline
\end{tabular}

${ }^{a}$ Minimum distance between points in a sample. 
distance $r$ of a given sample lie within a circle of area $\pi r^{2}$. For sample points within a distance $r$ of the edge of the field, a portion of the area of this circle no longer contributes to the expected number of pairs. This reduction in pairs causes the inflection point and asymptotic behavior of $\Phi(r)$ as $r$ increases (Fig. 2, inset). The exact correction due to edge effects is given by Ward and Ferrandino for the continuous case (35).

Estimating mean incidence and the sample variance. The first test of a sampling scheme is how well the statistics of the sample describe the population as a whole. Consider a sample containing $n$ unique individuals (without replacement) chosen from a population of $N$ individuals of which $I$ are infected. If every possible combination of choosing the sample from the population is equally likely to occur, then the probability that the sample contains $i$ infected individuals is simply the number of ways one can choose $n$ plants of which $i$ are infected divided by the total number of ways one can choose $n$ plants from the population regardless of their disease status. This ratio is called the hypergeometric distribution, $H(i, n, I, N)$, and is given by (pages 43-44 in literature citation 7)

$$
\begin{aligned}
& H(i, n, I, N)=\frac{\left(\begin{array}{l}
I \\
i
\end{array}\right)\left(\begin{array}{c}
N-I \\
n-i
\end{array}\right)}{\left(\begin{array}{c}
N \\
n
\end{array}\right)}=\frac{\left(\begin{array}{c}
n \\
i
\end{array}\right)\left(\begin{array}{c}
N-n \\
I-i
\end{array}\right)}{\left(\begin{array}{c}
N \\
I
\end{array}\right)} \\
& \text { where } \quad\left(\begin{array}{l}
A \\
B
\end{array}\right)=\frac{A !}{(A-B) ! \cdot B !} \text { so that } \\
& H(i, n, I, N)=\frac{n ! \cdot I ! \cdot(N-n) ! \cdot(N-I) !}{i ! \cdot(n-i) ! \cdot(I-i) ! \cdot(N-n-I+i) ! \cdot N !}
\end{aligned}
$$

Defining the fraction of the total population which is diseased as $p=I / N$, the expectation value or mean for $i, E_{H}(i)$, and variance, $\sigma_{H}{ }^{2}$, are given by (page 233 in literature citation 7)

$$
\begin{aligned}
& E_{H}(i)=\frac{n I}{N}=n p \\
& \sigma_{H}^{2}=\frac{n I}{N} \cdot\left(1-\frac{I}{N}\right) \cdot\left(1-\frac{n-1}{N-1}\right)=n p(1-p) \cdot\left(1-\frac{n-1}{N-1}\right)
\end{aligned}
$$

In the limit, as the number of samples becomes vanishingly small compared with the population as a whole $(n<<N)$, equation $3 \mathrm{~b}$ approaches the value $n p(1-p)$, which is the predicted variance for a binomial distribution. The hypergeometric equation (equation 2) is appropriate if either the sampling pattern or the epidemic exhibits complete spatial randomness. The symmetry between the nature of the two-dimensional sampling pattern and the spatial pattern of disease can be viewed as a double-marked process (30). In such a process we have a locus of points (a field of plants) of which a certain fraction are marked (say painted red) and then a second marking process is applied (say a painted green dot). If the two marking processes are independent, then the resultant number of doubly marked loci is given by the hypergeometric equation (equation 2). This is true whether the red painted loci are diseased plants and the green dot signifies a sampled plant or visa versa. This symmetry is reflected in the fact that the quantities $n$ and $I$ enter equation 2 in exactly the same fashion.

In order to understand the variance for aggregated sampling patterns or aggregated epidemics, one must examine the statistical behavior of a sum of random variates. From within a sample of size $n$, I define the random variable, $X_{k}$, to assume the values of 1 or 0 according to whether the $k$ th plant in the sample is diseased or healthy, respectively. The total number of infected plants in the sample, $i$, is given by

$$
i=\sum_{k=1}^{n} X_{k}
$$

Conceptually, this sampling process can be repeated many times using different realizations of the process that created the epidemic. Various statistics (means, variances, and covariances) can then be defined in terms of ensemble averages over all the realizations (pages 214-215 in literature citation 24, Appendix). In particular, the ensemble variance of $i, \sigma_{i}^{2}$ is

$$
\sigma_{i}^{2}=\sum_{k=1}^{n} \sigma_{X_{k}}^{2}+2 \cdot \sum_{j>k}^{n} \operatorname{cov}\left(X_{j}, X_{k}\right)
$$

where the second sum is over the covariances of all possible unique pairs of sampling points.

Stationarity. In order to proceed, one must now make the following assumptions concerning the uniformity or stationarity and the isotropy of the epidemic (page 53 in literature citation 4): (i) The ensemble means (denoted by angle brackets) of each and every sampling point is the same, i.e., for all $k,\left\langle X_{k}\right\rangle=\mu_{X}=p=$ $I / N$ and $\langle i>=n p$. (ii) The variance about the mean is independent of sampling point and equal to $\sigma_{X}^{2}=p(1-p)=\sigma_{B}^{2}$, the binomial variance. (iii) The conditional probability that point $X_{k}$ is infected, given that point $X_{j}$ is infected $\left[p_{k \mid j}=p\left(X_{j} \rightarrow X_{k}, r_{j k}=r\right)=g(r)\right]$ is a function of the distance between the two points, $r_{j k}=r$. This is the event-event probability density function (page 636 in literature citation 4) and is analogous to Ripley's $g$ function (30). Likewise, the covariance of two sampling points, $X_{j}$ and $X_{k}$, is a function of the distance between the two points, $r_{j k}, \operatorname{cov}\left(X_{j}, X_{k}\right)=\operatorname{cov}\left(r_{j k}\right)$. Given the above assumptions, equation $5^{\prime}$ becomes

$$
\sigma_{i}^{2}=n \sigma_{B}^{2}+2 \cdot \sum_{j>k}^{n} \operatorname{cov}\left(r_{j k}\right)
$$

The normalized relative error in mean incidence. Subtracting $n \sigma_{B}^{2}$ from both sides of equation 5 and dividing by this same term, one obtains

$$
\sum_{i}^{2} \frac{\left(\sigma_{i}^{2}-n \sigma_{B}^{2}\right)}{n \sigma_{B}^{2}}=2 \cdot \sum_{j>k}^{n} \frac{\operatorname{cov}\left(r_{j k}\right)}{n \sigma_{B}^{2}}=\frac{2}{n} \sum_{j>k}^{n} \rho\left(r_{j k}\right)
$$

where $\rho\left(r_{j k}\right)$ (Appendix) is the spatial correlation coefficient for sampling points a distance of $r_{j k}$ apart, and $\Sigma_{i}^{2}$ is the centered and normalized variance of the number of infected plants in the sample, $i$. With the assumptions of isotropy, stationarity, and homogeneity, equation 6 becomes

$$
\Sigma_{i}^{2}=\frac{2}{n} \sum_{\text {all possible }} \phi(r) \rho(r)
$$

The normalized variance of $i, \Sigma_{i}^{2}$, is a measure of the relative error in the mean incidence of the sample compared with a random sample. As an epidemic becomes more and more spatially random, we would expect both $\rho(r)$ and $\Sigma_{i}^{2}$ (equation 7) to become vanishingly small. The aforementioned symmetry between $I$ and $n$ suggests that equation 7 should also go to zero if the sampling pattern is random, whether or not spatial correlation is present. This fact suggests that a positive correlation within a certain distance $r$ must be cancelled out by negative correlation at larger distances when $\phi(r)$ in equation 7 is indicative of random sampling (Fig. 2).

Spatially based estimates of statistics. The means, variances, covariances, and correlation coefficients defined above and in the Appendix are all based on ensemble averages over multiple realizations of an epidemic. In reality, of course, an epidemiologist is faced with incomplete samplings of one realization of an epi- 
demic. In this case, the ability to estimate ensemble averages using spatial averages hinges on a crucial property of the spatial point process that resulted in the observed epidemic known as ergodicity (pages 53-58 in literature citation 4: pages 215-218 in literature citation 24). For simplicity, we will assume that the observed epidemic possesses this property and account for problems as they arise.

Once again, assume we have a sample of $n$ individuals chosen using a prescribed sampling scheme from a population of $N$ individuals of which $I$ are infected. Of the $n$ sampled individuals, $i$ are observed to be infected. We will estimate $p=I / N$, the probability that a certain individual is infected by the sample average (denoted by an overbar):

$$
p=<X>\approx \bar{X}=\frac{1}{n} \sum_{j=1}^{n} X_{j}=\frac{i}{n}
$$

where expressions to the left of the similarity sign, " $\approx$ ", are ensemble averages and expressions to the right are spatial averages. Equation 8 is a straightforward extension of the ensemble average to a spatial average. The estimation of second-order statistics using spatial averages involves the numbers of point pairs within a certain distance range ( $r \pm \Delta r / 2$; Appendix). These pairs of plants fall into three distinct categories: doubly infected, mixed infection (one plant healthy and one plant infected), and doubly healthy. All pertinent statistics can be expressed in terms of the numbers of plant pairs that fall into each of the above categories. Take for example the covariance of two sample points a distance $r$ apart, $\operatorname{cov}(r)$. By definition, for any two sampling points, $X_{j}$ and $X_{k}$, a distance $r$ apart:

$$
\operatorname{cov}(r)=\left\langle\left(X_{j}^{\alpha}-<X_{j}>\right) \cdot\left(X_{k}^{\alpha}-<X_{k}>\right)\right\rangle=<X_{j} \cdot X_{k}>-<X_{j}>\cdot<X_{k}>
$$

Consider the first term on the far right side of equation $9,\left\langle X_{j} \cdot X_{k}\right\rangle$, the mean of the product. This term represents the probability that the two plants located at sample points $X_{j}$ and $X_{k}$, a distance $r_{j k}=r$ apart, are simultaneously infected, $\left\langle X_{j} \cdot X_{k}\right\rangle$, and is usually estimated as

$$
\begin{gathered}
<X_{j} X_{k}>=\left.p \cdot g(r) \approx \overline{X_{j} X_{k}}\right|_{r-\Delta r / 2<r j k<r+\Delta r / 2}= \\
\frac{1}{\phi(r)} \sum_{j=1}^{n} \sum_{k>j}^{n} X_{j} X_{k} \cdot \delta\left(r_{j k}-r\right)=\frac{\phi_{i i}(r)}{\phi(r)}
\end{gathered}
$$

where $\delta\left(r_{j k}-r\right)$ is the Kronecker delta (24), which is unity if its argument is zero, and zero otherwise; $\phi(r)$ is the total number of point pairs; and $\phi_{i i}(r)$ is the number of doubly infected point pairs a distance of $r \pm \Delta r / 2$ apart (Appendix, equation A8). The estimation of the second term on the far right of equation 9, $\left\langle X_{j}\right\rangle \cdot\left\langle X_{k}\right\rangle$, depends on the evaluation of the mean using spatial averages.

$$
\begin{aligned}
<X_{j}> & \approx \frac{1}{2 \cdot \phi(r)} \sum_{j=1}^{n} \sum_{k>j}^{n}\left\{2 \cdot X_{j} X_{k}+\left(X_{j}-X_{k}\right)^{2}\right\} \delta\left(r_{j k}-r\right)=P(r) \\
& =\frac{\left[2 \cdot \phi_{i i}(r)+\phi_{i h}(r)\right]}{2 \cdot \phi(r)}
\end{aligned}
$$

where $\phi_{i h}(r)$ is the number of mixed point pairs a distance $r$ apart (Appendix, equation A6). The final form of equation 11 reflects the fact that at a distance $r$ there are $\phi(r)$ unique pairs representing $2 \cdot \phi(r)$ plants of which $2 \cdot \phi_{i i}(r)+\phi_{i h}(r)$ are infected. Since $g(r)$ is defined in terms of the conditional probability that a plant a distance $r$ away from an infected plant is also infected, it can be directly estimated as the number of infected plants divided by the total number of plants located at a distance $r$ from each and every infected plant, i.e.,

$$
g(r) \approx G(r)=\frac{2 \cdot \phi_{i i}(r)}{2 \cdot \phi_{i i}(r)+\phi_{i h}(r)}
$$

Note that the number of doubly infected pairs enters equation 11 twice, once for each infected plant of the pair. Combining equations $11,12, \mathrm{~A} 4$, and $\mathrm{A} 5$, the covariance, $\operatorname{cov}(r)$, the spatial correlation coefficient, $\rho(r)$, and the variogram, $2 \gamma(r)(4)$, can be approximated in terms of $G(r)$ and $P(r)$, which in turn can be directly estimated from the numbers of plant-pairs (equations 11 and 12):

$$
\begin{aligned}
& \operatorname{cov}(r) \approx P(r) \cdot(G(r)-P(r)) \\
& \rho(r) \approx \frac{G^{(r)}-P(r)}{1-P(r)} \\
& 2 \gamma(r) \approx \frac{\phi_{i h}(r)}{\phi(r)}=2 P(r) \cdot(1-G(r))
\end{aligned}
$$

where both $G(r)$ and $P(r)$ are calculated using a subsample of plant-pairs composed only of sampled plants that have neighboring plants lying within the pertinent distance range $(r \pm \Delta r / 2)$. Typically, in spatial correlation analysis, the value $P(r)$ in the covariance (equation 13a) and the correlation coefficient (equation $13 \mathrm{~b})$ are based on the entire sample (4) (i.e., $P(r)$ is replaced by $p)$. As will be shown later, the use of subsample means significantly reduces the variance of the estimated value of the correlation coefficient and can be thought of as a sort of "moving average method." In calculating the above estimates, the size of the window about distance $r$ (i.e., $\pm \Delta r / 2$; Appendix) is somewhat arbitrary. Cressie (4) recommends making $\Delta r$ large enough so that at least 30 plant-pairs fall into each category. For the second order statistics evaluated using equation 13 , this simple rule of thumb is inadequate at low values of disease incidence.

The correlation coefficient, $\rho$, in terms of a $\chi^{2}$ test. One of the advantages of using equation $13 \mathrm{~b}$ to estimate the value of the correlation coefficient, $\rho(r)$, is that confidence limits can be placed on the resultant value using a $\chi^{2}$ analysis (Table 3; Appendix). A minimum detectable value of the correlation coefficient, $\rho_{\text {crit }}(r)$, can be derived from equation A11 in terms of the total number of plant pairs in the distance range, $\phi(r)$ :

$$
\begin{aligned}
& \rho_{\text {crit }}(r)=\sqrt{\frac{\chi^{2}\left(\alpha_{\text {crit }}\right)}{\phi(r)}}=1.645 \cdot(\phi(r))^{-0.5} \text { for } \alpha_{\text {crit }}=0.1 \\
& \text { or } \\
& \phi(r)=2.705 /\left(\rho_{\text {crit }}(r)\right)^{2}
\end{aligned}
$$

Thus, using equation 14, at least 272 plant pairs are needed to detect a correlation coefficient as small as 0.1 . The validity of the

TABLE 3. Number of plant pairs $(\phi)$ in a $2 \times 2$ contingency table for application of Fisher's exact test (Appendix) ${ }^{\mathrm{a}}$

\begin{tabular}{lccc}
\hline Plant $1 \backslash$ plant 2 & Infected & Healthy & Total \\
\hline Infected & $\phi_{i i}$ & $\phi_{i h} / 2$ & $\phi_{i i}+\phi_{i h} / 2$ \\
Healthy & $\phi_{i h} / 2$ & $\phi-\phi_{i i}-\phi_{i h}$ & $\phi-\phi_{i i}-\phi_{i h} / 2$ \\
Total & $\phi_{i i}+\phi_{i h} / 2$ & $\phi-\phi_{i i}-\phi_{i h} / 2$ & $\phi$ \\
\hline
\end{tabular}

a The nonsubscripted "phi" $(\phi)$ represents the total number of plant pairs and subscripts indicate whether both (ii) or only one (ih) of the plants in a plant pair are infected. 
above $\chi^{2}$ analysis is subject to the constraint that expectation values for the various plant pair numbers in the contingency table (Table 3) are all above a certain minimal value (15) (i.e., $\phi(r)[P(r)]^{2}>5$ and $\left.\phi(r)[1-P(r)]^{2}>5\right)$. At very low disease incidence, this constraint can be a problem. Note that the expectation value for the number of doubly infected plant pairs, $\phi_{i i}$, is equal to the product of the total number of plant pairs, $\phi(r)$, and the square of the infection probability $p^{2}$. For small values of $p$, an inordinately large number of plant pairs may be necessary to ensure the validity of the $\chi^{2}$ approach. For example, if $p$ is $<0.05$, then the number of plant pairs must exceed 2,000 $\left(5 / p^{2}\right)$. Such a large number of plant pairs within a certain distance range may not be practical due to economical or time constraints, and may be geometrically impossible due to the presentiment of the plants in the field.

This information about correlation provided by the above analysis will, of necessity, inflate the variance of the estimated mean disease incidence. Using equation 7 , we see that even one critical detection of a value of $\rho_{\text {crit }}(r)=0.1$ with 272 plant pairs results in an increase of the normalized variance of $12.3 \%$ $(=100 \% \cdot 2 \cdot 272 \cdot 0.1 / 441)$. Significant correlation at a number of distances can easily double the variance of the mean disease.

Testing the sampling schemes. In order to test the relative efficiency of the various sampling schemes, I first construct a series of spatial point patterns or "mock epidemics" that vary in the number of infected plants, $I$, the degree to which disease is aggregated, $\aleph$ (Appendix), and the length scale, $a$, over which this aggregation occurs. The latter two of these properties correspond to what Pielou calls the intensity and the granularity of the spatial pattern, respectively (28).

Consider a square field of $N=40,000$ host plants arranged in a square grid pattern with 200 plants on a side. Henceforth, unit distance shall be assumed to be the plant spacing. To avoid edge effects, samples will be confined to an inner square with 168 on a side containing $N_{A}$ plants $\left(N_{A}=168^{2}=28,224\right)$. This leaves an annular boundary region 16 plants wide containing $N_{B}$ plants $\left(N_{B}=\right.$ $\left.N-N_{A}=11,776\right)$. The plan is to randomly generate a fixed number of disease foci within the field. Then each of these foci produce infected plants according to a given radial density function, $D(r)$ of distance $r$ from the center of each focus (Appendix). The above is an example of a Neyman-Scott cluster process (page 661 in literature citation 4), which has been applied to problems ranging from hemacytometer data (25) to distributional problems in cosmology (26).

In order to evaluate the different sampling schemes, 1,000 simulated epidemics were run for each of the 100 combinations of four values for the number of infected plants, $I\left(2^{11}, 2^{12}, 2^{13}\right.$, and $\left.2^{14}\right)$, with five levels of focal aggregation $(\aleph=0.2,0.4,0.6,0.8$, and 1.0; Appendix), and five values for the dispersive length scale, $a=1,2,4,8$, and 16 . One realization for each of nine of these combinations $\left(I=2^{13} ; s=0.2,0.6\right.$, and $1.0 ; a=4,8$, and 16) is illustrated in Figure 3.

Observational inference and experimental data. Thus far, we have examined the nature of spatial point patterns through the eyes of an omniscient observer privy to the calculation of ensemble averages. An experimentalist is confronted with a single set of $n$ sample points of which $i$ are infected. Exactly what information does this data set convey? We would like to estimate mean disease incidence along with its uncertainty, and decide whether or not the infected plants are spatially aggregated. In addition, if spatial aggregation is present, we would like to describe its nature and magnitude in some way.

Obviously, the data provides an unbiased estimator for mean disease incidence $(I / N)$ given by $p=i / n$ (31; equation $3 a)$. The probability distribution of this estimator is completely specified if either the sampling of points or the epidemic itself exhibit complete spatial randomness (equations 2 and $3 \mathrm{~b}$ ). However, the error in this estimator is not immediately obtainable for nonrandom sampling patterns when the disease is spatially aggregated.

In order to determine whether or not the disease is distributed randomly, we must examine the population of sampled point pairs. Each sample point $j$ has a location $\left(x_{j}, y_{j}\right)$ in the field and a binary value of either 1 or $0\left(X_{j}\right)$ depending on whether or not the sampled point is infected. These $n$ samples can be used to construct $n(n-1) / 2$ unique point pairs $\left[\left(X_{j}, X_{k}\right) ; k>j\right]$, which by definition are a distance $r_{j k}=\left[\left(x_{j}-x_{k}\right)^{2}+\left(y_{j}-y_{k}\right)^{2}\right]^{1 / 2}$ apart. Each of these point pairs belong to one of three categories: either both plants are infected (ii), both are healthy $(h h)$, or the infection is mixed $(i h)$. The number of point pairs $(\phi(r))$ obtained from plants lying within a certain distance $\left(\left|r_{j k}-r\right|<\Delta r / 2\right)$ of each other can be apportioned into each of the above categories. The resultant population information can then be used in a $\chi^{2}$ analysis to determine if the spatial pattern of disease significantly deviates from random behavior (Table 3; equations 11, 12, 13b, 14, and A10), where

$$
\chi^{2}=\rho^{2}(r) \cdot \phi(r)
$$

The choice of the value for $\Delta r$ in the above analysis must be large enough to ensure that the expectation values for both doubly infected pairs and doubly healthy pairs, $P(r)^{2} \phi(r)$ and [1 $P(r)]^{2} \phi(r)$, respectively, each exceed a value of $5(15)$. Note that as the value of $\Delta r$ is increased, the total number of point pairs, $\phi(r)$, also increases, which increases the power of the analysis; however, spatial resolution will suffer. We must also take into consideration that, if equation 15 is applied over many distance ranges, the result is subject to error due to multiple comparisons and the Bonferroni correction will apply $(12,13)$.

In order to put the analyses on an equal footing for all the tested sampling schemes and to eliminate the multiple comparison problem, I propose using a $\chi^{2}$ analysis based on the cumulative pair probability distribution function, $\Phi(r)$ (Fig. 2). In this case, the total number of plant pairs can once again be apportioned among the three infection categories (i.e., $i i, i h$, and $h h$ ) and the value of $\chi^{2}$ is calculated using equation A12. Of course $\Phi(r)$ will increase with increasing $r$, but assuming that spatial correlation is a monotonically decreasing function of point pair distance, average spatial correlation (equation A12) decreases with increasing $r$. In practice, the value of $r$ can be adjusted to maximize the value of $\chi^{2}$. The relative ability of each sampling scheme in detecting focal aggregation can then be described by the detection efficiency, $\mathrm{DE}(\alpha)$, defined as the fraction of disease realizations that give a significant maximal $\chi^{2}$ at probability level $\alpha$ (equation A12). This method of analysis not only indicates whether or not significant aggregation is present, but also provides an initial estimate of the length scale, $r_{\chi}$, which is defined as the radius at which equation A12 reaches its maximum value. The value of $r_{\chi}$ is actually an estimate of the distance range within which spatial correlation is positive and thus tends to overestimate correlative length scale.

An alternative estimate for the length scale over which contagion is important can be obtained directly from the observed values of $\rho(r)$. For this analysis, $\Delta r$ was chosen to be one half the distance between plants so that minimal pooling occurred. The correlative length scale, $r_{\rho}$, was then defined by

$$
r_{\rho}=\frac{\int_{r=0}^{r_{\chi}} \rho(r) d r}{\operatorname{Max}\left[\rho(r) ; 0<r<r_{\chi}\right]}
$$

In practice, the integral in equation 16 was estimated using the trapezoid rule. In effect, $r_{\rho}$ represents the width of a "Top Hat" distribution whose height is equal to the maximum observed value of the correlation coefficient and whose area under the $\rho$ versus $r$ curve is equal to the estimate obtained from observation. 


\section{RESULTS}

Illustrative examples using ensemble averages. To demonstrate the aforementioned symmetry between the spatial patterns of sampled plants, $n$, and infected plants, $I$, two stochastic experiments were conducted. The first experiment involved the random sampling of five different mock epidemics for which $I=$ $8,192=2^{13}, p=0.29, s=1$, and $a=1,2,4,8$, and 16. Each of these five spatial patterns was sampled over 5,000 trial runs. The resultant frequency distributions for the observed $i$ are plotted in Figure 4A. In the second experiment, 8,192 infected plants were randomly placed in the field 5,000 times and sampled using four cluster patterns $(1 \times 1,2 \times 2,4 \times 4$, and $8 \times 8)$. The resultant frequency distributions for the observed $i$ are plotted in Figure 4B. As previously stated, the hypergeometric equation (equation 2) is appropriate in either case.

To examine the effect of cluster sampling of a spatially aggregated epidemic, 1,000 simulations of an epidemic with $I=8,192$, $s=0.8$, and $a=2$ were run and sampled using four cluster patterns $(1 \times 1,2 \times 2,4 \times 4$, and $8 \times 8)$. The resultant frequency distributions for $i$ are plotted in Figure 5 (left panel). The variance of $i$ for each sampling scheme increases with decreasing cluster size, and the uniform grid sampling scheme with the largest length scale results in a variance smaller than predicted by the hypergeometric distribution (equation $3 \mathrm{~b}$ ). These results indicate that equation 2 is no longer valid when both the epidemic and the sampling scheme exhibit spatial aggregation. In this more general case, the variance in $i, \sigma_{i}^{2}$, is governed by equations 5 to 7 and the interaction between the correlation coefficient, $\rho(r)$, and the point-pair distribution function, $\phi(r)$, becomes important (equation 7). The value of the correlation coefficient, $\rho(r)$, can be directly estimated using equations 11,12 , and $13 \mathrm{~b}$. This was done for 1,000 simulated epidemics using all of the above sampling schemes and the resultant average correlation coefficients are also plotted in Figure 5 (right panel). The theoretical value calculated using equation A9 is plotted in Figure 5 as a dashed line for comparison. The sampling scheme exhibiting the largest variance in $i$ (Fig. 5, $1 \times 1$ cluster) provides the most information about spatial correlation. The normalized variance of the mean $\left(\Sigma_{i}^{2}\right.$, equation 7$)$ is plotted in Figure 6 for five different sampling schemes for simulated epidemics ( 1,000 realizations) characterized by five different dispersal length scales ( $a=1,2,4,8$, and 16) at two values

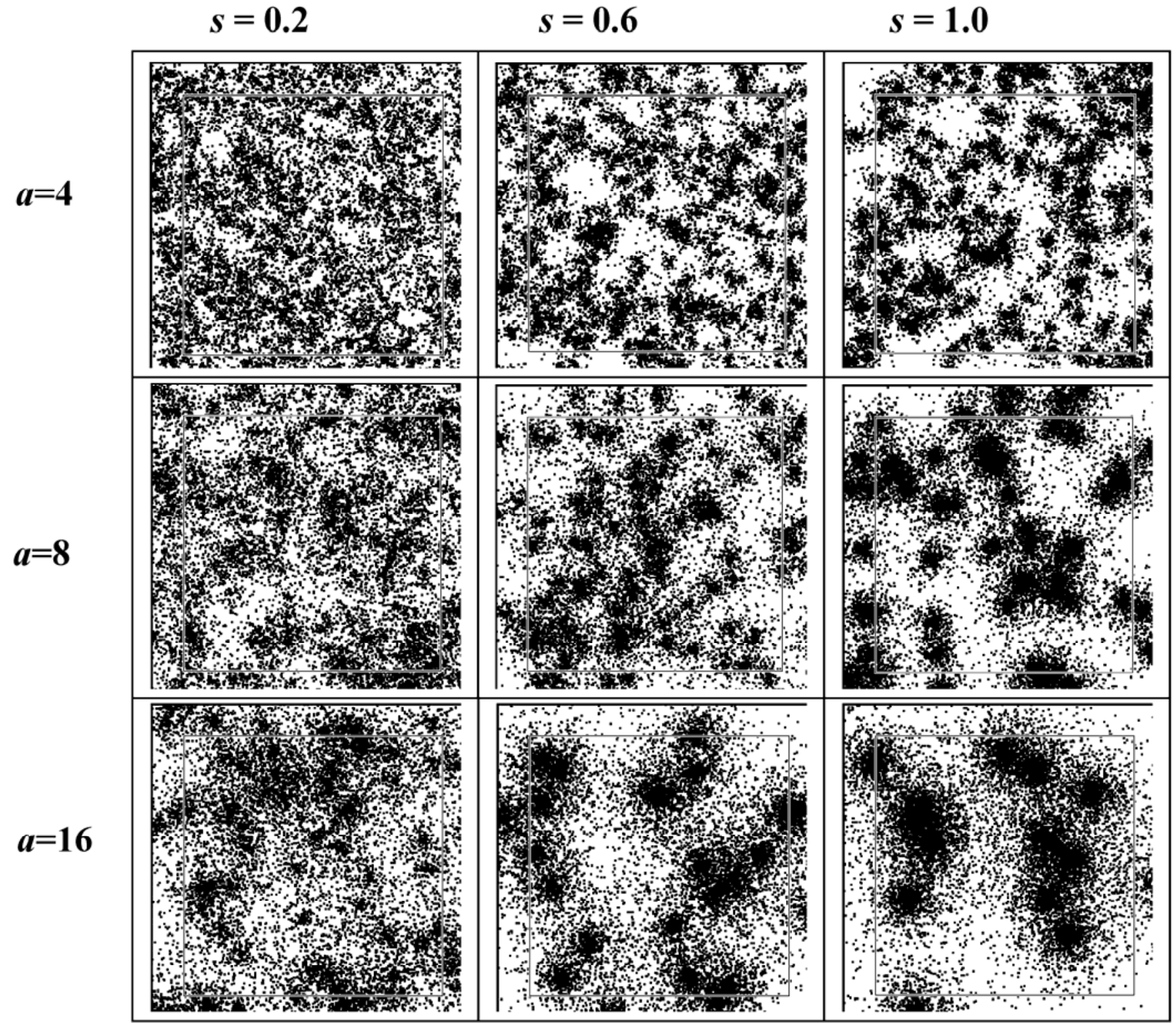

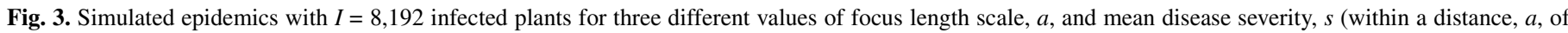
each focus). Each square represents a field with 200 plants on a side. Sampling is restricted to the inner square $(168 \times 168)$ to minimize edge effects. 
for the infection probability $(p=0.07$ and 0.59$)$. The net result is that at all but the smallest length scale $(a=1)$, the nested fractal design results in the largest variance. This is a direct result of the relation expressed in equations 5 to 7 between variance of the sum and the plant-pair weighted sum over the spatial correlations. The inflation of the variance of the mean for fractal sampling can be partially offset using a weighted average of the sample points as an estimator of mean incidence (Fig. 6, dashed line with open squares). The optimal value for the weight given to each sample point is obtained by regression analysis (page 20 in literature citation 4 ; 6).

Detection and quantification of aggregation. The effect of using subsample averages on the calculation of the estimated correlation coefficient (equation 13b) was investigated using a fractal sampling of 1,000 realizations for each of three spatially random epidemics with $p=0.07,0.29$, and 0.56 . The use of the subsampling approach (Fig. 7, filled symbols) considerably reduces the variance of the estimate when compared with the con- ventional use of the overall sample mean $(p=i / n)$ in correlation estimates (Fig. 7, open symbols). In addition, the resulting error is remarkably insensitive to disease incidence, $p$. The dotted line (Fig. 7, theory) corresponds to the inverse sample size.

In order to compare the relative ability of each sampling scheme in detecting focal aggregation, $\mathrm{DE}(0.05)$ is plotted versus focal radius, $a$, in Figure 8 for four different combinations of $s$ and $p(s=0.25$ and $1.0 ; p=0.073$ and 0.29$)$. The value of DE increases rapidly with increasing focal radius, $a$, and increases to a lesser extent with disease incidence, $p$, and focus saturation, $s$ (Fig. 8). As one might expect, focal aggregation is best detected by the sampling schemes that provide the most point pairs at smaller distance ranges (Fig. 8, cluster $[1 \times 1]$ and fractal).

The impact of sampling scheme on the correlative length scale, $r_{\rho}$ (equation 16), is shown in Figure 9 for fractal and random sampling patterns. The increased number of plant pairs less than 16 units apart for the fractal sampling scheme greatly enhances both the accuracy and precision of the estimate for correlative
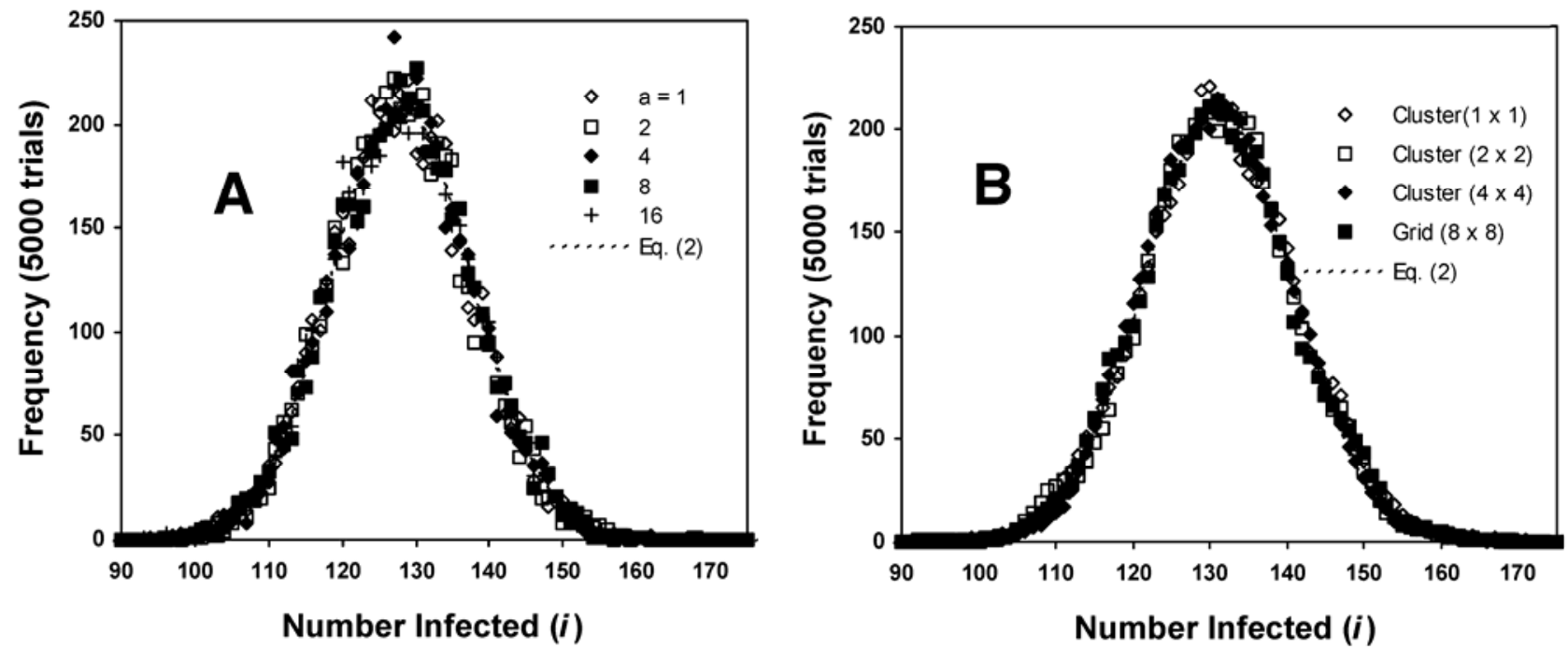

Fig. 4. Frequency distribution of the number of infected plants in a sample. A, Results for a random sample taken from 5,000 replicate simulations for each of five values of the dispersive length scale, $a(1,2,4,8$, and 16), with $I=8,192$ infected plants and disease severity within the focus $(s)=1$. B, Results from cluster samplings of 5,000 randomly selected epidemics with $I=8,192$.
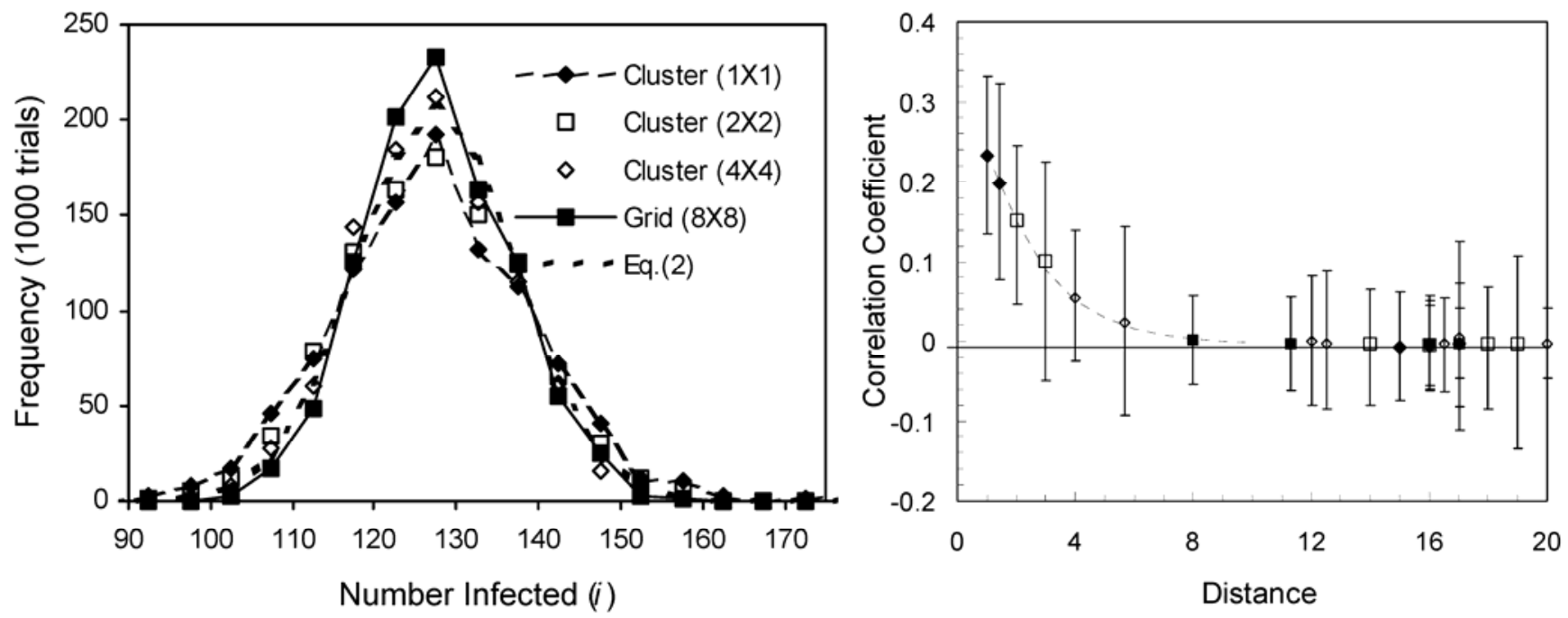

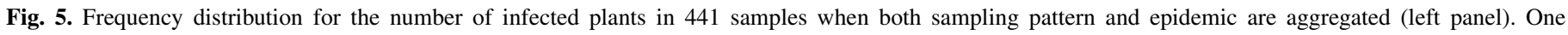

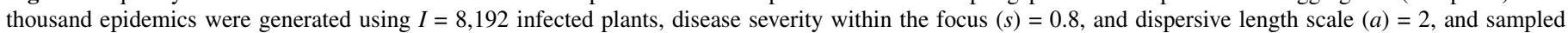

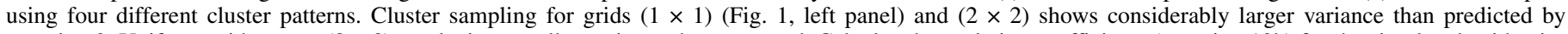

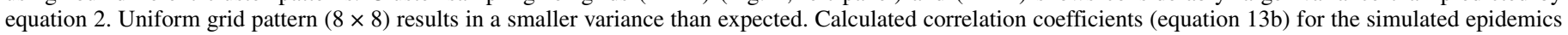

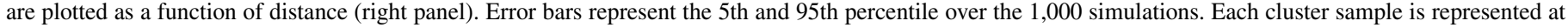

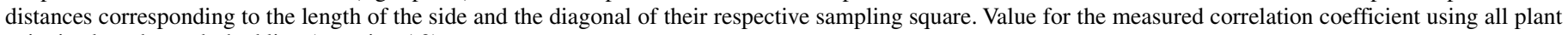
pairs is plotted as a dashed line (equation A9). 
length scale provided by equation 16 . The other sampling patterns that can be characterized by a specific length scale (cluster $[1 \times$ $1]$, cluster $[2 \times 2]$, cluster $[4 \times 4]$, and grid $[8 \times 8])$ give highly erratic estimates of the correlative length scale (Fig. 10).

Summary and conclusions. I have presented a new subsample-based estimate of the radial correlation function (equation $13 \mathrm{~b}$ ), which is shown to be equivalent to a $\chi^{2}$ analysis (equations A10 and A12). This analysis provides a method for direct calculation of confidence intervals about measured correlations. The cumulative form of the analysis (equation A12) allows for the unambiguous detection of significant aggregation (Fig. 8) free from the uncertainty brought about by multiple comparisons. In addi- tion, the analysis provides a measure for degree of aggregation (equation A12) and an estimate for the correlative length scale (equation 16).

This method was applied to 100 different stochastically generated mock epidemics varying over a factor of 8 in disease incidence $(p=0.073-0.59)$, a factor of 16 in dispersive length scale $(a=1-16)$, and a factor of 5 in focal saturation $(s=0.2-$ 1 ). One thousand realizations for each of these 100 epidemics were generated using a Neyman-Scott cluster process $(25,26)$. Each realization was sampled using six different sampling patterns, and the resulting data were analyzed using the subsamplebased correlation method.
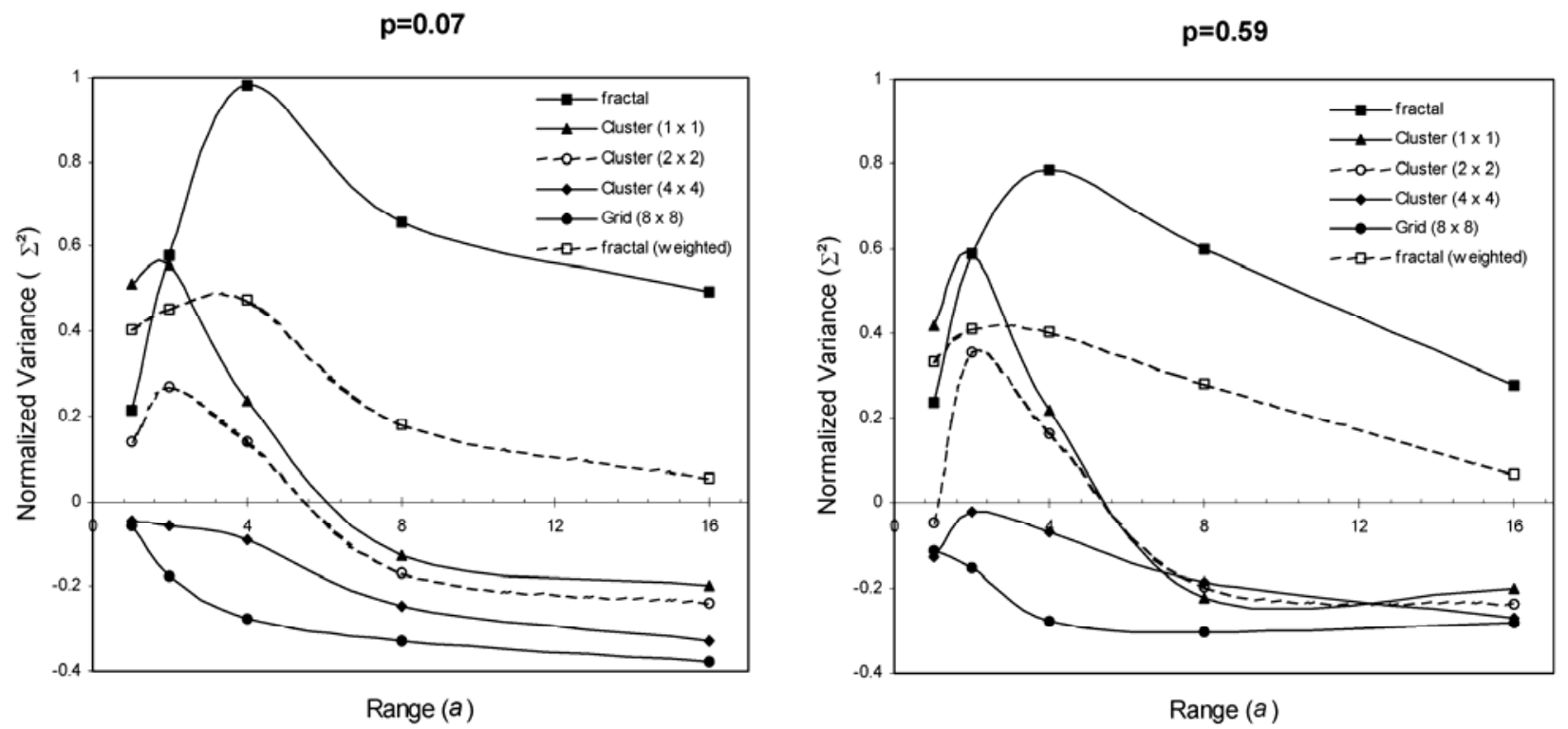

Fig. 6. Normalized variance of observed mean incidence $\left(\Sigma^{2}\right.$; equations 6 and 7) plotted versus dispersal length scale $(a)$ for the various sampling schemes illustrated in Figure 1. A value of zero corresponds to independent samples (equation 5). The grid sample always has the smallest variance and the fractal sample usually has the largest variance. The use of a weighted estimate for mean incidence results in a large decrease $(\approx 50 \%)$ in the variance of the estimated mean incidence for the fractal sample. $P=$ infection probability.

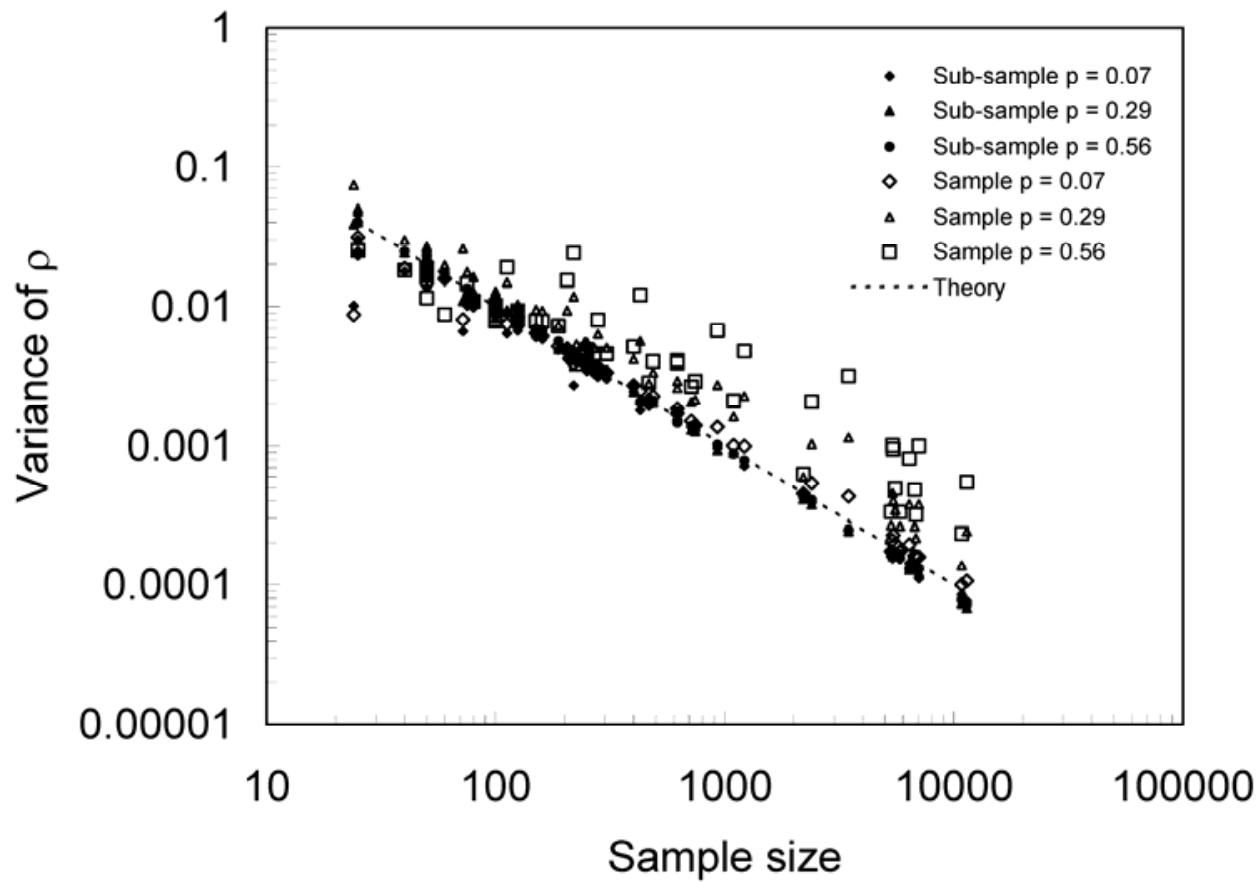

Fig. 7. Plot of the value of the variance of the calculated spatial correlation function, $\rho$, versus sample size, $n$, for infection probability $(p)=0.07,0.29$, and 0.56 . Variance was evaluated as the ensemble average over 1,000 realizations of a spatially random epidemic. Filled symbols (equation $13 \mathrm{~b}$ ) calculated using subsample averages for the probability of infection and open symbols use the more conventional sample average. 
As a result of this study, there are three general conclusions concerning the information obtained from various spatial sampling schemes. (i) Sampling patterns that maximize the distance between sampled points give the best estimates of disease incidence and the least information on spatial heterogeneity. (ii) Spatial sampling patterns, which yield spatial information on aggregation, inflate the variance of the resultant estimate of mean disease levels (equation 7). (iii) Maximum spatial information is obtained when many length scales are more or less equally represented in the sampling pattern. However, this information comes at the cost of inaccurate estimates of the mean disease level.

As in any complex system, the optimal sampling strategy must be a compromise. In this case, we must choose between clustered and evenly spaced sampling. This decision is critically dependent on the goals of the study in question, the implicit costs accrued in capital and labor both in acquiring and analyzing samples, and lastly, the prevalence of disease in the chosen sample. For cases where disease incidence is high enough for the estimation of spatial correlation, the nested fractal-based design described above provides the most reliable estimators of disease aggregation.

\section{APPENDIX}

Ensemble averages. From within a sample of size $n$, I define the random variable, $X_{k}$, to assume the values of 1 or 0 according to whether the $k$ th plant in the sample is diseased or healthy. The total number of infected plants in the sample, $i$, is given by the sum over the $X_{k}^{\text {''s: }}$

$$
i=\sum_{k=1}^{n} X_{k}
$$
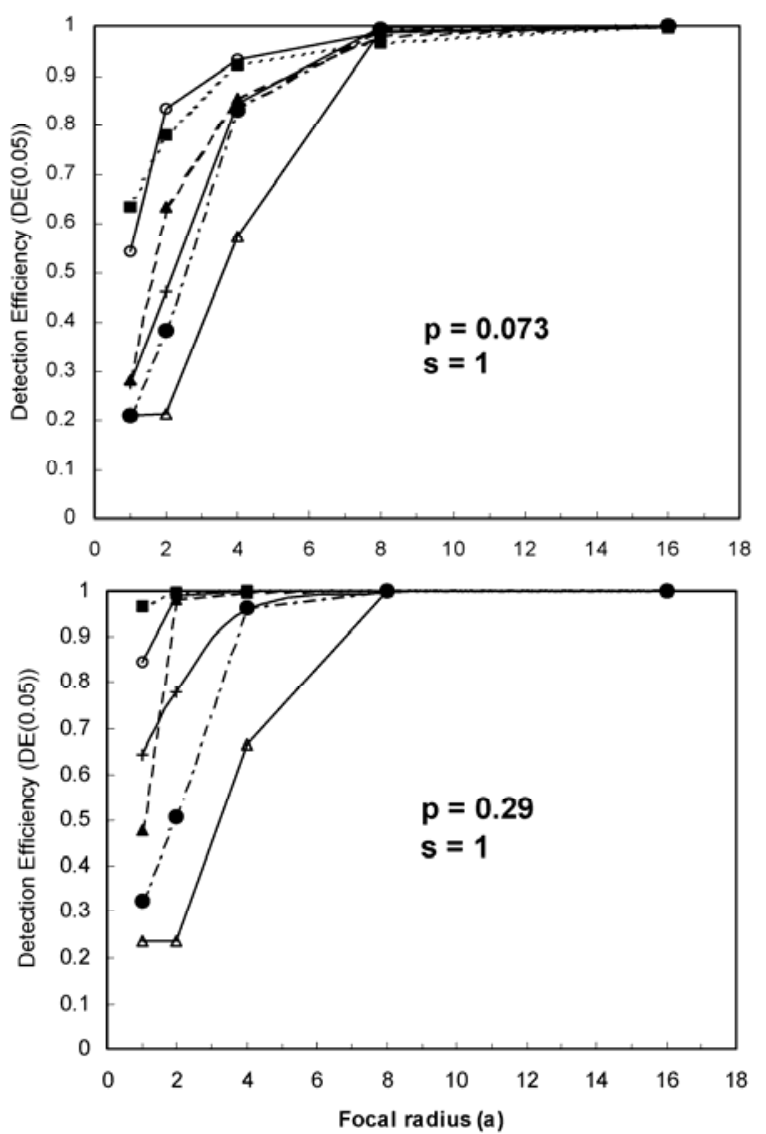

Suppose this sampling process is repeated $T$ times and that the results of the $\alpha$ th trial are denoted by a superscripted $\alpha$, then the mean value (ensemble average denoted by angle brackets " $<>$ " placed around the quantity in question) of the $X_{k}^{\alpha}$ 's, $\left\langle X_{k}\right\rangle$, the $i^{\alpha}$ s, $<i>$, and the variance about these means, $\sigma_{X}{ }^{2}$ and $\sigma_{i}^{2}$, are given by

$<X_{k}>=\frac{\sum_{\alpha=1}^{T} X_{k}^{\alpha}}{T}$
$\sigma_{X_{k}}^{2}=\frac{\sum_{\alpha=1}^{T}\left(X_{k}^{\alpha}-<X_{k}>\right)^{2}}{T}$

$<i>=\frac{\sum_{\alpha=1}^{T} i^{\alpha}}{T}=\frac{\sum_{\alpha=1}^{T} \sum_{k=1}^{n} X_{k}^{\alpha}}{T}=\sum_{k=1}^{n}<X_{k}>$

$\sigma_{i}^{2}=\frac{\sum_{\alpha=1}^{T}\left(i^{\alpha}-<i>\right)^{2}}{T}=\frac{\sum_{\alpha=1}^{T}\left(\sum_{k=1}^{n} X_{k}^{\alpha}-<i>\right)^{2}}{T}=\frac{\sum_{\alpha=1}^{T}\left(\sum_{k=1}^{n}\left(X_{k}^{\alpha}-<X_{k}>\right)\right)^{2}}{T}$

Note that the ensemble average of $X_{k}$ in equation A1a is simply the probability that the plant located at the $k$ th sampling location is infected, $p_{k}$. Define the ensemble covariance of $X_{j}$ and $X_{k}$, $\operatorname{cov}\left(X_{j}, X_{k}\right)$, as

$$
\begin{aligned}
& \operatorname{cov}\left(X_{j}, X_{k}\right)=\frac{\sum_{\alpha=1}^{T}\left(X_{j}^{\alpha}-<X_{j}>\right) \cdot\left(X_{k}^{\alpha}-<X_{k}>\right)}{T}= \\
& <X_{j}^{\alpha} X_{k}^{\alpha}>-<X_{j}><X_{k}>
\end{aligned}
$$
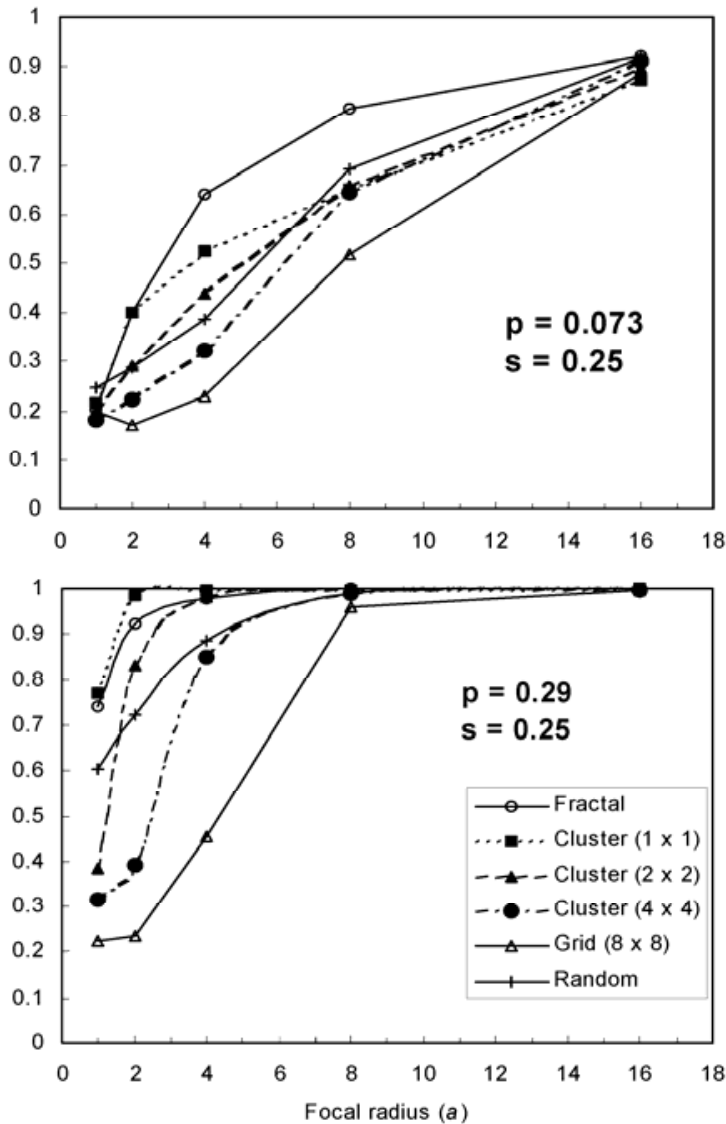

Fig. 8. Plots of detection efficiency $(\operatorname{DE}(\alpha) ; \alpha=0.05)$ versus focal radius, $a$, obtained from the six sampling patterns (described in text). Sampling patterns that have a large number of plant pairs at small distance scales (fractal and cluster $[1 \times 1]$ ) are most efficient in detecting aggregation. $p=$ disease incidence; $s=$ disease severity within the focus. 
Since this is a binary system, the ensemble average of the first product on the far right of equation A3 is simply the probability that the plants located at sample points $j$ and $k$ are both simultaneously infected (i.e., $X_{j}=1$ and $X_{k}=1$ ). This simultaneous probability can be expressed as the product of the probability that point $j$ is infected, $p_{j}$, and the conditional probability that point $k$ is also infected, given that point $j$ is infected, $p_{k \mid j}$. Likewise, the last product in equation $\mathrm{A} 3$ becomes $p_{j} \cdot p_{k}$ and equation $\mathrm{A} 3$ can be rewritten as

$$
\operatorname{cov}\left(X_{k}, X_{j}\right)=p_{j} p_{k \mid j}-p_{j} p_{k}
$$
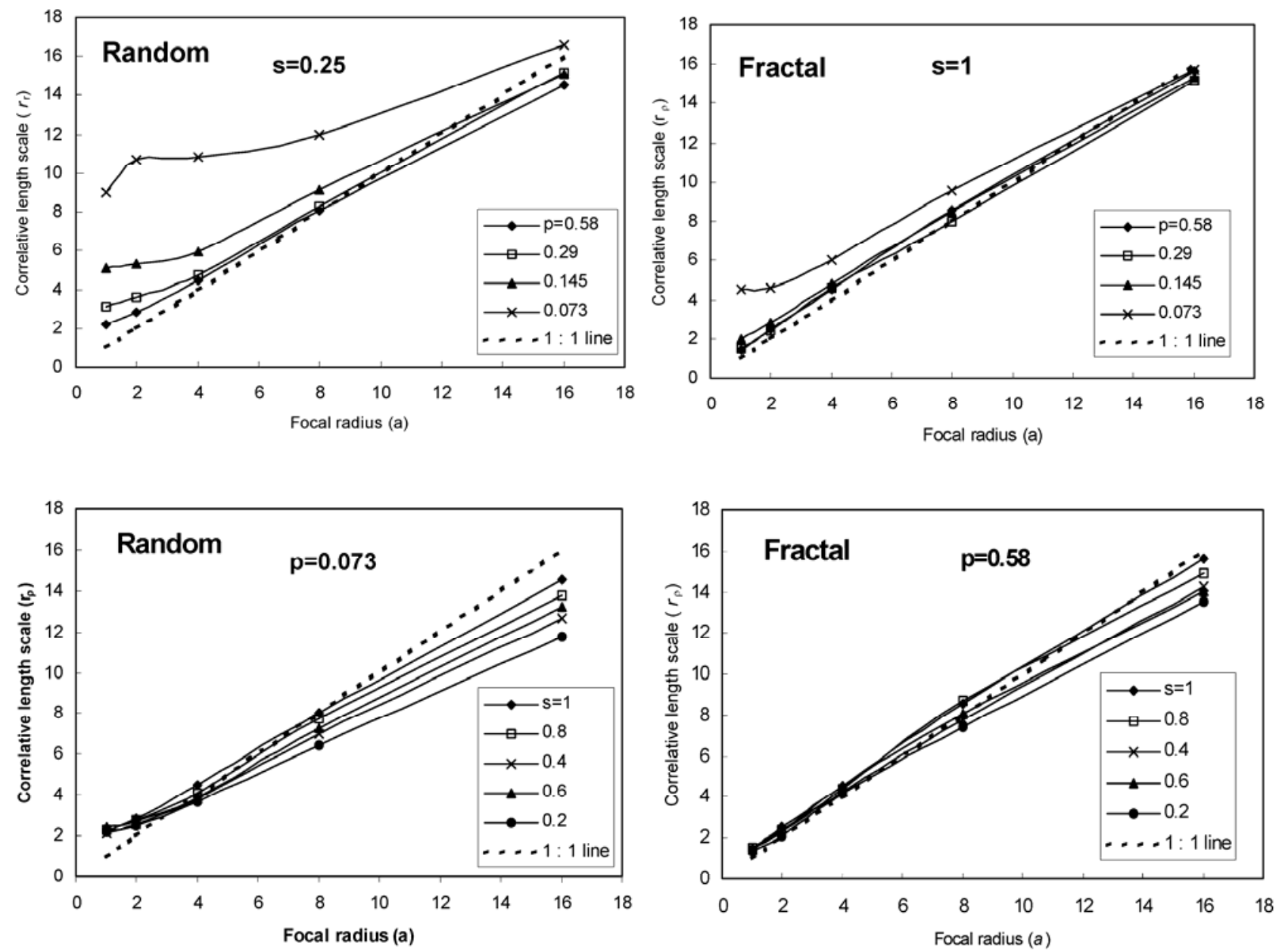

Fig. 9. Comparison of the median value of 1,000 realizations for the correlative length scale, $r_{\rho}$ (equation 16), obtained using either a random or a fractal sampling pattern. $p=$ disease incidence; $s=$ disease severity within the focus.
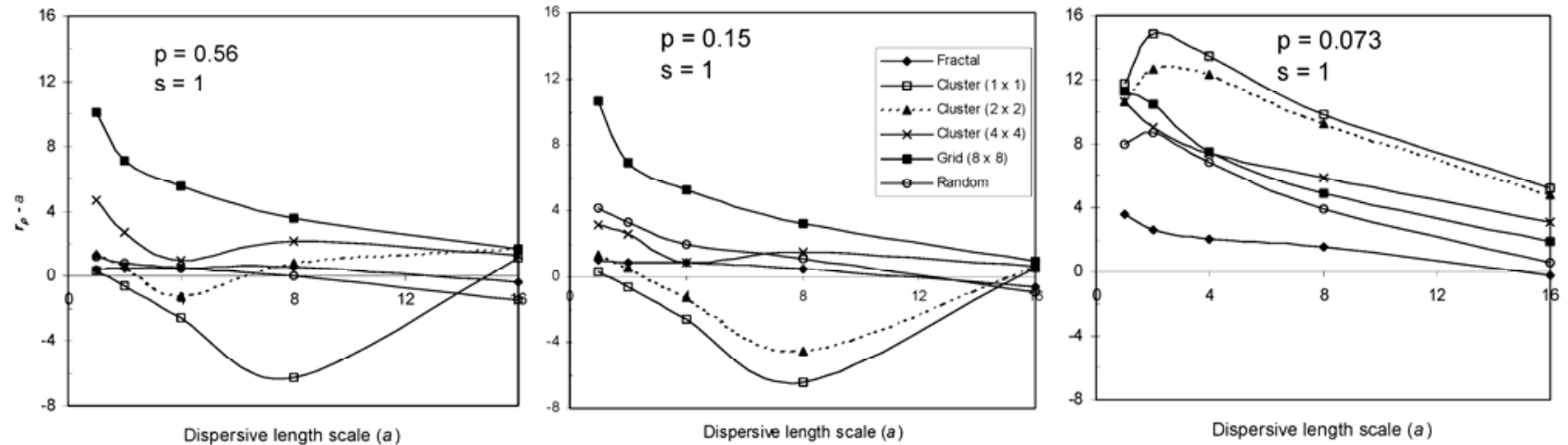

Fig. 10. Median difference between the correlative length scale, $r_{\rho}$ (equation 16), and the dispersive length scale, $a$, for 1,000 realizations of a simulated epidemic plotted versus $a$ for different sampling schemes. $p=$ disease incidence; $s=$ disease severity within the focus. 
Under the assumptions of stationarity (described previously), assuming that points $j$ and $k$ are a distance $r$ apart, equations A4 and A5 simplify to

$$
\operatorname{cov}(r)=p \cdot g(r)-p^{2}
$$

and

$$
\rho(r)=\frac{\operatorname{cov}(r)}{\sigma_{B}^{2}}=\frac{p \cdot g(r)-p^{2}}{p \cdot(1-p)}=\frac{g(r)-p}{1-p}
$$

Spatial point pairs. The estimation of equations A3 through A5 using spatial averages presents a problem. As a start, one might consider the double sum over all possible pairs of different sampled plants a distance $r$ apart. Since there might not be enough pairs at a specified distance, we choose a tolerance region such that $\left|r_{j k}-r\right|<\Delta r / 2$ (page 70 in literature citation 4). Depending on the sampling scheme, there will be $\phi(r)$ unique pairs of sampled plants within a distance range $r \pm \Delta r / 2$, of which a certain number, $\phi_{i i}(r)$, will be doubly infected, a number, $\phi_{h h}(r)$, will be composed of two healthy plants, and a number, $\phi_{i h}(r)=$ $\phi_{h i}(r)$, will be composed of mixed pairs, defined as follows:

$$
\begin{aligned}
& \phi(r)=\sum_{j=1}^{n} \sum_{k>j}^{n} \delta\left(r_{j k}-r\right) \\
& \phi_{i i}(r)=\sum_{j=1}^{n} \sum_{k>j}^{n} X_{j} X_{k} \delta\left(r_{j k}-r\right) \\
& \phi_{h h}(r)=\sum_{j=1}^{n} \sum_{k>j}^{n}\left(1-X_{j}\right) \cdot\left(1-X_{k}\right) \delta\left(r_{j k}-r\right) \\
& \phi_{i h}(r)=\phi_{h i}(r)=\sum_{j=1}^{n} \sum_{k>j}^{n}\left(X_{j}-X_{k}\right)^{2} \delta\left(r_{j k}-r\right) \\
& \text { where } \quad \delta\left(r_{j k}-r\right)=\left\{\begin{array}{ll}
1 & \text { if }\left|r_{j k}-r\right| \leq \Delta r / 2 \\
0 & \text { if }\left|r_{j k}-r\right|>\Delta r / 2
\end{array}\right\}
\end{aligned}
$$

It is not clear how to express the above point pairs as a simple inner product of two well-defined vectors in analogy with equation A3. The problem is that each sampling point may enter once, more than once, or not at all, depending on how many neighboring points it has at the prescribed distance. In addition, each sampling point enters as both $X_{j}$ and $X_{k}$ in a redundant fashion, one pair for each of its neighbors which are a distance $r$ away. However, the spatial analogy of the far right side of equation A3, $\left\langle X_{j}^{\alpha} \cdot X_{k}^{\alpha}\right\rangle-\left\langle X_{j}^{\alpha}\right\rangle \cdot\left\langle X_{k}^{\alpha}\right\rangle$, which is the ensemble average of the product minus the product of the ensemble averages, is easily obtained.

The correlation coefficient $\rho(r)$ (equations A7 and 13b) can then be expressed totally in terms of the point pair counts over the pertinent distance scale using equations 10 and 11 :

$$
\rho(r)=\frac{\phi(r) \phi_{i i}(r)-\left(\phi_{i i}(r)+\phi_{i h}(r) / 2\right)^{2}}{\left(\phi_{i i}(r)+\phi_{i h}(r) / 2\right)\left(\phi(r)-\phi_{i i}(r)-\phi_{i h}(r) / 2\right)}
$$

Now consider the $2 \times 2$ contingency table shown in Table 3 . The $\chi^{2}$ value for such a table is given by (15)

$$
\chi^{2}=\frac{\left[\phi(r) \phi_{i i}(r)-\left(\phi_{i i}(r)+\phi_{i h}(r) / 2\right)^{2}\right]^{2} \phi(r)}{\left(\phi_{i i}(r)+\phi_{i h}(r) / 2\right)^{2}\left(\phi(r)-\phi_{i i}(r)-\phi_{i h}(r) / 2\right)^{2}}
$$

A simple comparison of equations A9 and A10 yields

$$
\chi^{2}=\rho^{2}(r) \cdot \phi(r)
$$

Equation A11 can be used to calculate confidence limits and critical values for estimated values (equation 13b) of the correlation coefficient, $\rho(r)$, assuming a chi-squared distribution with one degree of freedom. The same arguments can be used to develop a $\chi^{2}$ analysis based on the cumulative pair probability distribution function, $\Phi(r)$, defined as the fraction of unique point pairs less than a distance $r$ apart. The result is

$$
\begin{aligned}
& \chi^{2}=\bar{\rho}^{2}(r) \Phi(r) \frac{n(n-1)}{2}= \\
& \frac{\left[\Phi(r) \Phi_{i i}(r)-\left(\Phi_{i i}(r)+\Phi_{i h}(r) / 2\right)^{2}\right]^{2} \Phi(r)}{\left(\Phi_{i i}(r)+\Phi_{i h}(r) / 2\right)^{2}\left(\Phi(r)-\Phi_{i i}(r)-\Phi_{i h}(r) / 2\right)^{2}} \cdot \frac{n(n-1)}{2}
\end{aligned}
$$

where the overbar on the "rho", $\bar{\rho}(r)$, indicates average correlation for distances less than $r, n$ is the number of samples, and $n(n-$ 1) $/ 2$ is the total number of unique sample point pairs. The use of equation A12, instead of multiple applications of equation A10 at various distances and values of $\Delta r$, helps to eliminate the need for Bonferroni corrections due the multiple comparisons $(12,13)$.

Simulated epidemics. Spatial patterns of infected plants (mock epidemics) were constructed stochastically using a Neyman-Scott cluster process (page 661 in literature citation 4). The first step is to randomly generate a fixed number of disease foci within the field. Each of these foci is then used to generate infective offspring, which are dispersed according to a given radial density function, $D(r)$ of distance $r$ from the center of each focus, to eventually produce infected plants. For the simulations that follow, I chose the Bessel distribution suggested by van den Bosch et al. (33):

$$
D(r)=\frac{1}{2 \pi r a} e^{-r / a}
$$

where $a$ is the characteristic length scale of dispersal.

Consider a square field of $N=40,000$ host plants arranged in a square grid pattern with 200 plants on a side. Henceforth, unit distance shall be assumed to be the plant spacing. To avoid edge effects, samples will be confined to an inner square (region A) with 168 on a side containing $N_{A}$ plants $\left(N_{A}=168^{2}=28,224\right)$. This leaves an annular boundary region (region B) 16 plants wide containing $N_{B}$ plants $\left(N_{B}=N-N_{A}=11,776\right)$. The spatial behavior of the mock epidemic is determined by the following three parameters: (i) $I$, the total number of infecteds (marked plants) within region $\mathrm{A}$; (ii) $F$, the number of randomly placed foci in the entire field (region $\mathrm{A}+$ region $\mathrm{B}$ ); and (iii) $a$, the mean radius of each focus in terms of plant spacing.

First, the central coordinates of each focus is chosen independently, each with a uniform distribution over the field. Note the coordinates of the $j$ th foci $\left(x_{j}, y_{j}\right)$ are not forced to fall at the center of a plant but can take on fractional values. For each focus in turn, a distance, $r$, is independently selected such that the probability of $r>R$ is given by

$$
P(r>R)=e^{-R / a}
$$

The algorithm used is to first generate a pseudorandom number, $\psi$, between 0 and 1 and then set

$$
r=a \cdot[-\ln (\psi)]
$$

Next, an angle $\theta$ is chosen from a uniform distribution between $0^{\circ}$ and $360^{\circ}$ and the coordinates of an offspring point $(X, Y)$ are calculated using the following prescription

$$
\begin{aligned}
& X=x_{j}+r \cdot \cos \theta \\
& Y=y_{j}+r \cdot \sin \theta
\end{aligned}
$$

The net result of the above procedure is to produce a locus of points $(X, Y)$ about the center of each focus with a radial probability density function given by the above Bessel distribution 
(equation A13). If the specified coordinate $(X, Y)$ does not fall within region $\mathrm{A}$, no action is taken and we proceed to the next focus of disease. If the specified coordinate $(X, Y)$ is within region A, we follow one of two prescriptions depending on whether a binary (plant $=$ healthy or diseased) or an intensity-based (severity $\mathrm{S}=$ number of lesions per plant) description of disease is desired. For a binary epidemic, if the plant nearest to $(X, Y)$ is already infected, no action is taken; otherwise, the plant nearest to that coordinate is infected. For an intensity-based epidemic, the severity of the plant nearest to $(X, Y)$ is incremented by one. In either case, the process continues cyclically for each focus in turn until the specified number of plants, $I$, within region A are infected.

In the discussion that follows, it will be useful to define a number of calculated parameters that describe various aspects of a given epidemic. The mean number of infecteds per focus is given by

$$
i_{F}=\frac{I \cdot\left(N_{A}+N_{B}\right)}{F \cdot N_{A}}
$$

For the epidemic of Figure 5, $i_{F}=336.6$. The mean area of a focus is given by $\pi a^{2}$, which roughly corresponds to the mean number of plants affected by a focus. Thus, a natural measure of the intensity of a binary epidemic is given by the average disease severity, $s$, within a distance, $a$, of each disease focus:

$$
s=\frac{i_{F}}{\pi a^{2}}=\frac{I \cdot\left(N_{A}+N_{B}\right)}{\pi a^{2} \cdot F \cdot N_{A}}
$$

For the epidemic of Figure 5, $s=0.419$. There are some natural restrictions to the value of $i_{F}$, the mean number of infecteds per focus. If $i_{F}$ is exactly one, then the epidemic does not exhibit any contagion and the epidemic approaches complete spatial randomness. In addition, if $s$ exceeds unity $\left(i_{F}>\pi a^{2}\right)$, then the focus becomes saturated and will have length scale larger than $a$. Thus, in the binary simulations that follow $1<i_{F}<\pi a^{2}$, so that $1 /\left(\pi a^{2}\right)<$ $s<1$. Defining $p$ as the probability of infection within region A $\left(p=I / N_{A}\right)$, if $s$ is $<p$, then the density within a focus is less than the mean density and the epidemic is not aggregated around foci but rather between foci. This kind of expulsion pattern may be applicable to tree densities in forests around large trees $(30,35)$. With this in mind, I define the intensity of focal aggregation, $\aleph$, by

$$
\aleph=\frac{s-p}{1-p}
$$

which varies from 0 to 1 as foci become more and more saturated.

For the epidemic of Figure $5, \aleph=0.272, p=0.202$, and as above $s=0.419$. Thus, within region $\mathrm{A}$, the incidence of disease is about $20 \%$. However, if we restrict our attention to the plants within 16 plant units of the disease foci, the incidence of disease is about $42 \%$.

In order to evaluate the different sampling schemes, 1,000 simulated epidemics were run for each of the 100 combinations of four values of $I\left(2^{11}, 2^{12}, 2^{13}\right.$, and $\left.2^{14}\right)$ with five values of $\aleph(0.2$, $0.4,0.6,0.8$, and 1.0) and five values of $a(1,2,4,8$, and 16). One realization for each of nine of these combinations $\left(I=2^{13} ; s=0.2\right.$, 0.6 , and $1.0 ; a=4,8$, and 16) is illustrated in Figure 3.

\section{LITERATURE CITED}

1. Clayton, M. K., and Hudelson, B. D. 1995. Confidence intervals for autocorrelations based on cyclic samples. J. Am. Stat. Assoc. 90:753-757.

2. Clinger, W., and Van Ness, J. W. 1976. On unequally spaced time points in a time series. Ann. Stat. 4:736-745.

3. Cochran, W. G. 1977. Sampling Techniques. John Wiley \& Sons, New York.
4. Cressie, N. 1991. Statistics for Spatial Data. John Wiley \& Sons, New York.

5. De Vries, P. G. 1986. Sampling Theory for Forest Inventory. SpringerVerlag, Berlin.

6. Draper, N. R., and Smith, N. 1966. Applied Regression Analysis. John Wiley \& Sons, New York.

7. Feller, W. 1970. An Introduction to Probability Theory and Its Application, Volume 1. 2nd ed. John Wiley \& Sons, New York.

8. Ferrandino, F. J. 1989. Spatial and temporal variation of a defoliating plant disease and reduction in yield. Agric. For. Meteorol. 47:273-290.

9. Ferrandino, F. J. 1989. A distribution-free method for estimating the effect of aggregated plant damage. Phytopathology 79:1229-1232.

10. Ferrandino, F. J. 1993. Dispersive epidemic waves: I. Focus expansion within a linear planting. Phytopathology 83:795-802.

11. Ferrandino, F. J. 1996. The length scale of disease spread: Fact or artifact of experimental geometry. Phytopathology 86:806-811.

12. Ferrandino, F. J. 1996. Two-dimensional distance class analysis of disease-incidence data: Problems and possible solutions. Phytopathology 86:685-691.

13. Ferrandino, F. J. 1998. Past nonrandomness and aggregation to spatial correlation: 2DCORR, a new approach for discrete data. Phytopathology 88:84-91.

14. Ferrandino, F. J., and Elmer, W. H. 1991. Reduction in tomato yield due to Septoria leaf spot. Plant Dis. 76:208-211.

15. Fisher, R. A., and Yates, F. 1948. Statistical Tables for Biological, Agricultural and Medical Research. Hafner Publishing Company, New York.

16. Horsfall, J. G., and Barratt, R. W. 1945. An improved rating system for measuring plant disease. (Abstr.) Phytopathology 35:655.

17. Hudelson, B. D., Clayton, M. K., Smith, K. P., and Upper, C. D. 1997. Detection and description of spatial patterns of bacterial brown spot of snap beans using cyclic samples. Phytopathology 87:33-34.

18. Hughes, G. 1988. Spatial heterogeneity in crop loss assessment models. Phytopathology 78:883-884.

19. Hughes, G., and Madden, L. V. 1993. Using the beta-binomial distribution to describe aggregated patterns of disease incidence. Phytopathology 83:759-763.

20. Hughes, G., Madden, L. V., and Munkvold, G. P. 1996. Cluster sampling for disease incidence. Phytopathology 86:132-137.

21. Hughes, G., McRoberts, N., Madden, L. V., and Gottwald, T. R. 1997. Relationships between disease incidence at two levels in a spatial hierarchy. Phytopathology 87:542-550.

22. Karandinos, M. G. 1976. Optimum sample size and comments on some published formulae. Bull. Entomol. Soc. Am. 22:417-421.

23. Legendre, P., and Fortin, M. J. 1989. Spatial pattern and ecological analysis. Vegatio 80:107-138.

24. Monin, A. S., and Yaglom, A. M. 1973. Statistical Fluid Mechanics: Mechanics of Turbulence. MIT Press, Cambridge, MA.

25. Neyman, J. 1939. On a new class of "contagious" distributions applicable in entomology and bacteriology. Ann. Math. Stat. 10:35-57.

26. Neyman, J., and Scott, E. L. 1958. Statistical approach to problems in cosmology. J. R. Stat. Soc. B 20:1-29.

27. Olea, R. A. 1984. Sampling design optimization for spatial functions. J. Int. Assoc. Math. Geol. 16:369-392.

28. Pielou, E. C. 1969. An Introduction to Mathematical Ecology. John Wiley \& Sons, New York.

29. Ridout, M. S., and Xu, X.-M. 2000. Relationships between several quadrat-based statistical measures used to characterize spatial aspects of disease incidence data. Phytopathology 90:568-575.

30. Ripley, B. D. 1977. Modelling spatial patterns. J. R. Stat. Soc. B 39:172212.

31. Stuart, A. 1984. The Ideas of Sampling. Statistical Monograph No. 4, Griffin and Co., Wycombe, UK.

32. Turechek, W. W., Ellis, M. A., and Madden, L. V. 2001. Sequential sampling for incidence of Phomopsis leaf blight of strawberry. Phytopathology 91:336-347.

33. van den Bosch, F., Zadoks, J. C., and Metz, J. A. J. 1988. Focus expansion in plant disease. I: The constant rate of focus expansion. Phytopathology 78:54-58.

34. Wagonner, P. E., and Rich, S. 1981. Lesion distribution, multiple infection, and the logistic increase of plant disease. Proc. Natl. Acad. Sci. USA 78:3292-3295.

35. Ward, J. S., and Ferrandino, F. J. 1998. New derivation reduces bias and increases power of Ripley's L index. Ecol. Modelling 116:225-236. 